Carmona Sáez, P., Parra Martínez, J. y Gomariz Vicente, V. (2021). Participación de las familias de alumnado con apoyos y atenciones diferentes: un estudio en un contexto multicultural. Revista de Investigación Educativa, 39(1), 49-69 DOI: http://dx.doi.org/10.6018/rie.386551

\title{
Participación de las familias de alumnado con apoyos $y$ atenciones diferentes: un estudio en un contexto multicultural'.
}

\section{Participation of families of students with different supports and attention: a study in a multicultural context}

\author{
Patricia Carmona Sáez, Joaquín Parra Martínez y María Ángeles Gomariz Vicente \\ Departamento de Métodos de Investigación y Diagnóstico en Educación Universidad de Murcia
}

\begin{abstract}
Resumen
Familia y escuela colaborando en la educación del alumnado es un deseo y un derecho reconocido por la comunidad educativa. Se aborda, en un contexto multicultural en el sureste español, centrando la mirada de las familias cuyos hijos de Educación Infantil, Primaria y Secundaria Obligatoria reciben apoyo o atención diferentes. Busca conocer su implicación en la educación de los hijos y si existen diferencias respecto a la implicación de familias cuando sus hijos reciben, o no, apoyo o atención diferentes y en función de variables sociodemográficas. Han participado 3639 familias, se aplicó un cuestionario de elevada fiabilidad, con siete dimensiones de la participación. Las familias cuyos descendientes reciben apoyo o atención diferentes, atendiendo al modelo teórico que se defiende, se comunican más frecuentemente con los centros, muestran un elevado sentimiento de pertenencia, y valoran su participación formal en la escuela a través de órganos colegiados de manera más intensa.

Palabras clave: Participación de la familia; necesidades educativas; Educación Infantil; Educación Primaria; Educación Secundaria Obligatoria.
\end{abstract}

Correspondencia: Joaquín Parra Martínez, jparra@um.es, Facultad de Educación, Universidad de Murcia, 30100, Murcia.

1 El trabajo es parte de un Proyecto financiado por convocatoria pública por un ministerio del Gobierno de España (I+D+I, MINECO). 


\begin{abstract}
The collaboration of schools and families in students' education is both the wish and a recognized right within the educational community. This paper looks at families of children in Preschool and Primary and Secondary Education with varying degrees of care and support, within a multicultural context in the South-East of Spain. It seeks to know their degree of involvement in the children's education and whether this involvement changes with respect to their children's differing levels of care and support across sociodemographic variables. 3639 families have participated in this study by completing a questionnaire of high reliability that reflects seven dimensions of the families' aforementioned involvement. Families whose children receive different support or attention, according to our theoretical model, communicate more frequently with centers, show a high sense of belonging, and more intensely value their formal participation in schools through collegiate committees.

Keywords: Family involvement; educational needs; Early Childhood Education; Primary Education, Secondary Education.
\end{abstract}

\title{
Introducción
}

Para que la educación sea un proyecto común en torno al desarrollo de la persona, debe existir una participación de todos los actores que intervienen en el proceso de enseñanza-aprendizaje con el fin de que la consecuencia de ese desarrollo repercuta de forma positiva en el avance de la sociedad. Desde la escuela y la familia se deben propiciar aprendizajes que ayuden, a los alumnos ${ }^{2}$ e hijos, en un futuro a afrontar los cambios que se vayan produciendo en la sociedad, además de conocimiento para la mejora de la misma. El reto es conseguir que familia y escuela se unan para compartir un proyecto en torno al alumno con el fin de que este reciba la mejor educación posible.

La participación de las familias en la educación es un derecho básico en el caso de España. Este derecho se recoge en el gran número de leyes dispuestas por los poderes públicos, haciéndose realidad en los centros escolares regulados por estas. En la Constitución española se recogen los siguientes apartados del Artículo 27: Artículo 27.5 “Los poderes públicos garantizan el derecho de todos a la educación, mediante una programación general de la enseñanza, con participación efectiva de todos los sectores afectados y la creación de centros docentes." Artículo 27.7 "Los profesores, los padres y, en su caso, los alumnos intervendrán en el control y gestión de todos los centros sostenidos por la Administración con fondos públicos, en los términos que la ley establezca." Ley Orgánica para la Mejora de la Calidad Educativa 8/2013 (2013) dice en el apartado segundo del preámbulo lo siguiente:

La realidad familiar en general, y en particular en el ámbito de su relación con la educación, está experimentando profundos cambios. Son necesarios canales y hábitos que nos permitan restaurar el equilibrio y la fortaleza de las relaciones entre alumnos y alumnas, familias y escuelas. Las familias son

\footnotetext{
2 Utilizaremos el masculino como genérico como recomienda la RAE.
} 
las primeras responsables de la educación de sus hijos y por ello el sistema educativo tiene que contar con la familia y confiar en sus decisiones.

Entendemos la participación de la familia en la escuela como la manera de interaccionar los diferentes agentes de la comunidad educativa entre sí, para comunicarse, coordinarse y cooperar sobre la educación de sus hijos (Egido, 2014; Parra, García-Sanz, Gomariz y Hernández-Prados, 2014a).

Los centros educativos tienen un papel decisivo a la hora de informar a las familias sobre las diferentes formas de implicación y participación de estas en los centros escolares. Las familias, informadas, podrán conocer y advertir a los centros sobre cuáles son sus necesidades de comunicación y participación en los centros. Según lo recogido en la Ley Orgánica de Educación 2/2006 (2006) en el artículo 118.3, "Las administraciones educativas fomentarán, en el ámbito de su competencia, el ejercicio efectivo de la participación del alumnado, profesorado, familias y personal de administración y servicios en los centros educativos", además en su artículo 118.3 nos indica que "A fin de hacer efectiva la corresponsabilidad entre el profesorado y las familias en la educación de sus hijos, las Administraciones educativas aportarán medidas que promuevan e incentiven la colaboración efectiva entre la familia y la escuela". La Ley Orgánica para la Mejora de la Calidad Educativa 8/2013 (2013) en su artículo 72 nos dice que "Los padres y los alumnos y alumnas podrán participar en el funcionamiento de los centros a través de sus asociaciones. Las Administraciones educativas favorecerán la información y la formación dirigida a ellos". Podemos decir que la familia puede y la escuela debe facilitar la participación para que esta se lleve a cabo de forma efectiva, siendo los beneficios de esta relación bidireccional múltiples, respecto al alumnado, a las propias familias, al profesorado o al clima del propio centro educativo.

Evidencias empíricas recogidas en varios trabajos nos aportan conclusiones respecto a su influencia en el rendimiento académico (Castro, Expósito, Lizasoain, López y Navarro, 2014; Cox, 2005), respecto al desarrollo socioafectivo (Saracostti, Lara, Martella, Miranda, Miranda-Zapata y Reininger, 2019) o su influencia en la reducción del absentismo escolar o la mejora del clima de convivencia y el bullying (Chang-Hun, y JuyoungSong, 2012), mostrando que el rendimiento académico y el desarrollo personal de los alumnos mejora de forma moderada con la participación de las familias en los centros. Garreta (2015) afirma que se desarrollan actitudes positivas por parte de los padres y un mayor nivel de satisfacción al sentirse parte del centro escolar. Otros autores, aportan la idea de que el profesorado que mantiene una actitud positiva hacia la participación en los centros de las familias consigue que conozcan mejor cuáles son sus expectativas y por tanto que éstas estén más receptivas. Darling-Hammond (2000) en su estudio nos ofrece datos sobre los beneficios que aportan las familias al propio funcionamiento del centro educativo.

Pero las formas o vías de participación, incluso los niveles de implicación de las familias en los centros, pueden ser muy diversos, tal y como propone Epstein (2002), quien nos ayuda a diferenciar entre seis tipos o niveles de participación familiar. En primer lugar, sitúa la implicación en la crianza y el cuidado de los hijos (paternalistamaternalista), las familias crean un clima para el mejor desarrollo del niño. En segundo lugar, encontramos la participación comunicativa (comunicativo) de las familias, estas 
intentan crear un sistema de intercambio de información con respecto a los niños. En tercer lugar, posiciona la participación activa (voluntariado) de ayuda dentro del aula y del centro creándose así un sentimiento de pertenencia y colaboración directa en el centro escolar del niño. En cuarto lugar, emplaza la participación de la familia en el aprendizaje (aprendizaje) en casa, el cual en la mayoría de los casos se limita en que los niños realicen las tareas escolares, y en muchos de estos casos se le proporciona al niño una ayuda externa (profesor particular, academias de apoyo) para lograr la ejecución de dichas tareas. En quinto lugar, ubica la participación en los órganos de gobierno del centro (modelo decisorio) como las asociaciones de madres y padres de alumnos (AMPA), el Consejo Escolar (CE) y las diferentes comisiones que existan en el centro, de esta forma las familias se involucran de una forma directa en ciertos aspectos de la dirección de la formación que se lleva a cabo en el centro del niño. Y en sexto y último lugar, hallamos la participación de la familia en la propia comunidad (comunitario), la unión de familia y centro escolar aporta una mejora del sentimiento de pertenencia haciendo que las familias participen de algo que consideran parte de ellos: la comunidad. La participación de las familias en la vida de los centros, compartiendo objetivos con el profesorado, no solo influye en el rendimiento académico, no solo genera cohesión en el propio centro, propicia a la vez involucramiento social como ciudadanos.

En este trabajo, partimos del modelo teórico basado en siete dimensiones defendido por algunos autores (Hernández-Prados, García-Sanz, Parra y Gomariz, 2017; Hernández-Prados, García-Sanz, Gomariz y Parra, 2019; Gomariz, Parra, García-Sanz y Hernández-Prados, 2019), como evolución de los de Epstein (2002) y Vogels (2002), quienes proponen la atención a: Comunicación con el centro, Participación en actividades organizadas por el centro, Sentimiento de pertenencia de las familias al centro, Participación a través de las Asociaciones de Madres y Padres del Alumnado y el Consejo Escolar del centro, Implicación educativa de las familias desde el hogar, Formación a familias y Participación comunitaria. Un modelo en el que se incorporan los distintos niveles de implicación (desde el no conocimiento de actividades o acciones, conocimiento, asistencia, a la organización y gestión de la participación familiar), así como el papel clave del profesorado como favorecedor de dicha implicación de las familias.

Por otra parte, diferentes estudios nos señalan que las interacciones y reuniones entre las familias y los docentes son mayores cuando existen dificultades que afectan al alumno ya sean de aprendizaje, socialización o asociadas a una discapacidad. Propicia una mejora en el clima de convivencia en el centro. Aunque los resultados de las investigaciones son divergentes, existen trabajos que defiende que las familias de alumnado con necesidades educativas especiales tienden a participar de manera más activa en los centros escolares, con el propósito de dar respuesta adecuada a las dificultades de sus hijos (Alomar, 2006).

En esta línea, en su análisis meta-analítico, Castro et al. (2014, p.95), nos dicen que parece "verosímil pensar que [...] la participación en la escuela por parte de los padres, se da especialmente cuando el estudiante presenta algún tipo de dificultad". El análisis realizado sobre la implicación de las familias en los CE de los centros, realizado por Parra, García-Sanz, Gomariz y Hernández-Prados (2014b) aporta datos relevantes al analizar la participación según la enseñanza, el porcentaje de participación de las familias en los centros específicos de Educación Especial es mayor al resto de centros. Esto 
nos hace plantearnos preguntas sobre si existen o no diferencias de participación de las familias en los centros según tengan o no los niños algún tipo de necesidad educativa, de qué tipo es esa participación y en qué momentos se dan. Robledo y García-Sánchez (2014) insisten en la necesidad de continuar investigando en variables familiares dinámicas, tales como la implicación educativa familiar, temática abordada en este trabajo.

Teniendo en cuenta que el proceso de escolarización del alumnado con Necesidades Específicas de Apoyo Educativo (NEAE) en algunas ocasiones se encuentra con el riesgo de que el alumno no pueda seguir el ritmo de sus compañeros y podría desembocar en una situación de rendimiento disfuncional. El potencial educativo de cada alumno reside en las oportunidades que se le ofrecen para adquirir las competencias necesarias para su desarrollo. En este tipo de casos, es aún más importante que exista comunicación, cooperación y coordinación entre las familias y los centros escolares, para que ambas partes conozcan y trabajen en conjunto para atender las dificultades de los alumnos con NEAE. La escuela inclusiva debe, en cierto modo, asegurar que todos los alumnos y sus familias, participen del proceso enseñanza aprendizaje como un miembro más de la comunidad (Hernández-Prados, Gomariz, Parra y García-Sanz, 2016). En cualquiera de los casos se ha de tener en cuenta sus características intrínsecas, como individuo no como parte de una categoría que clasifique y divida en grupos diferentes, estigmatización incluida (Pascale, Carrión Martínez y Fernández Martínez, 2017; Peñafiel, 2012).

Hasta ahora la mayoría de autores que han realizado investigaciones sobre el tema de la relación familia-escuela de niños con dificultades se han centrado en los niños diagnosticados con algún tipo de discapacidad. Vemos necesaria la ampliación del "objeto" de estudio a las familias de niños que presenten algún tipo de necesidad específica de apoyo educativo que peculiarice el proceso de enseñanza-aprendizaje y su desarrollo personal a lo largo de sus años de escolaridad. Dado que nos encontramos inmersos en el camino hacia el aprendizaje inclusivo, se precisa el conocimiento de cuáles son las características que presentan las familias de los niños con NEAE en diferentes dimensiones. Cuál es el tipo de comunicación que se crea desde la familia y la escuela y si esta presenta ciertos aspectos que la caracterizan.

\section{Método}

\section{Objetivos}

A partir de todo lo anterior, en este trabajo nos proponemos conocer cómo participan las familias con hijos que presentan necesidades específicas de apoyo educativo en la educación escolar y si existen diferencias entre su participación en los centros y el resto de familias cuyos hijos están escolarizados en Educación Infantil, Primaria y Secundaria Obligatoria, en un contexto multicultural situado en una región del sureste de España. Este propósito, atendiendo a nuestro marco teórico, se concreta en los siguientes objetivos específicos:

1. Describir el grado de participación de las familias en la educación escolar, en relación a las dimensiones: comunicación con el centro, participación en actividades del centro, sentido de pertenencia, implicación en AMPA y CE, implicación desde el hogar, participación comunitaria y en la formación de las propias familias, 
distinguiendo entre las familias con hijos que presentan necesidades específicas de apoyo educativo y aquellas cuyos hijos no presentan dichas necesidades.

2. Analizar la existencia de diferencias estadísticamente significativas en la participación de las familias en la educación escolar en función de la presencia de hijos con necesidades específicas de apoyo educativo, respecto a cada una de las dimensiones exploradas.

3. Estudiar las diferencias estadísticamente significativas en dicha participación familiar, según la procedencia nacional de los progenitores, y si su hijo posee o no NEAE.

\section{Población y Muestra}

Se procedió mediante muestreo intencional en el que se invitó a toda la población de familias con hijos escolarizados entre la etapa de Educación Infantil hasta la de Educación Secundaria Obligatoria, ambas incluidas, pertenecientes a 14 centros educativos (doce de titularidad pública y dos de concertada) situados en un contexto multicultural, en el que aproximadamente el $30 \%$ de la población es de origen no español, predominando la procedencia del norte de África y una colonia hindú, compuesto por seis núcleos de población (Región de Murcia, España). La población invitada se corresponde con 6768 estudiantes escolarizados en dichas etapas y sus respectivas familias. Tras el proceso de recogida y depuración de la información, se obtuvo una muestra real o productora de datos de 3639 familias informantes, de las cuales 232 señalan que sus hijos presentan algún tipo de NEAE.

Tal y como recoge la Tabla 1, han participado 2930 (80.4\%) familias de centros públicos, $643(17.7 \%)$ familias de centros concertados. Han participado 802 (22.0\%) familias con hijos en Educación Infantil, 2080 (57.1\%) familias con hijos en Educación Primaria y 672 (18.5\%) familias con hijos en la Educación Secundaria Obligatoria, de las cuales 2180 padres (59.8\%) y 2188 madres (60\%) son de nacionalidad española y 1348 padres (37\%) y 1379 madres $(37.8 \%)$ son de variadas nacionalidades extranjeras (nacionalidad padre: latinoamericano, 17.1\%; país europeo, 7.7\%; asiático, 4.3\%; Magreb, 69.2\%; subsahariano, $0.9 \%$; nacionalidad madre: latinoamericana, $19.6 \%$; país europeo, 7.5\%; asiático, 4.1\%; Magreb, 67.3\%; subsahariano, $0.9 \%$ ). El resto de variables recogidas aportan los porcentajes que se observan en la Tabla 1.

Tabla 1.

Características de la muestra participante. Frecuencias y porcentajes.

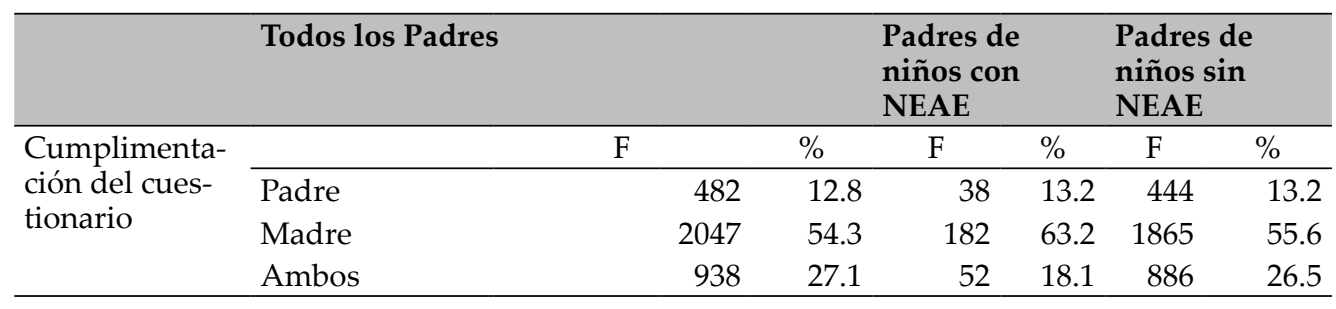




\begin{tabular}{|c|c|c|c|c|c|c|c|}
\hline \multirow[b]{2}{*}{ Edad } & \multicolumn{3}{|l|}{ Todos los Padres } & \multicolumn{2}{|c|}{$\begin{array}{l}\text { Padres de } \\
\text { niños con } \\
\text { NEAE } \\
\end{array}$} & \multicolumn{2}{|c|}{$\begin{array}{l}\text { Padres de } \\
\text { niños sin } \\
\text { NEAE } \\
\end{array}$} \\
\hline & Padre & & & & & & \\
\hline & De 31 a 40 & 1224 & 33.6 & 88 & 30.6 & 1136 & 33.8 \\
\hline & De 41 a 50 & 1843 & 50.6 & 150 & 52.1 & 1693 & 50.4 \\
\hline & Más de 51 & 322 & 8.8 & 24 & 8.3 & 298 & 8.9 \\
\hline & Madre & & & & & & \\
\hline & De 20 a 30 & 361 & 9.9 & 12 & 4.2 & 349 & 10.4 \\
\hline & De 31 a 40 & 1779 & 48.8 & 135 & 46.9 & 1644 & 49.0 \\
\hline & De 41 a 50 & 1332 & 36.6 & 121 & 42.0 & 1211 & 36.1 \\
\hline \multirow[t]{16}{*}{ Estudios } & Padre & & & & & & \\
\hline & Sin estudios & 478 & 13.1 & 23 & 8.0 & 455 & 13.6 \\
\hline & Primaria & 875 & 24.0 & 75 & 26.0 & 800 & 23.8 \\
\hline & Secundaria & 758 & 20.8 & 54 & 18.8 & 704 & 21.0 \\
\hline & $\begin{array}{l}\text { Bachillerato o } \\
\text { FP Medio }\end{array}$ & 708 & 19.4 & 58 & 20.1 & 650 & 19.4 \\
\hline & $\begin{array}{l}\text { FP superior o } \\
\text { Diplomatura }\end{array}$ & 338 & 9.3 & 24 & 8.3 & 314 & 9.4 \\
\hline & $\begin{array}{l}\text { Licenciatura, } \\
\text { grado o similar }\end{array}$ & 255 & 7.0 & 20 & 6.9 & 235 & 7.0 \\
\hline & Doctorado & 9 & .2 & .0 & .0 & 9 & .3 \\
\hline & Madre & & & & & & \\
\hline & Sin estudios & 413 & 11.3 & 16 & 5.6 & 397 & 11.8 \\
\hline & Primaria & 714 & 20.3 & 63 & 21.9 & 678 & 20.2 \\
\hline & Secundaria & 779 & 21.4 & 66 & 22.9 & 713 & 21.2 \\
\hline & $\begin{array}{l}\text { Bachillerato o } \\
\text { FP Medio }\end{array}$ & 704 & 19.3 & 68 & 23.6 & 636 & 19.0 \\
\hline & $\begin{array}{l}\text { FP superior o } \\
\text { Diplomatura }\end{array}$ & 575 & 15.8 & 49 & 17.0 & 526 & 15.7 \\
\hline & $\begin{array}{l}\text { Licenciatura, } \\
\text { grado o similar }\end{array}$ & 294 & 8.1 & 15 & 5.2 & 279 & 8.3 \\
\hline & Doctorado & 14 & .2 & .0 & .0 & 14 & .4 \\
\hline \multirow[t]{8}{*}{ Ocupación } & Padre & & & & & & \\
\hline & $\begin{array}{l}\text { Propietario pequeño ne- } \\
\text { gocio }\end{array}$ & 338 & 9.3 & 30 & 10.4 & 308 & 9.2 \\
\hline & $\begin{array}{l}\text { Trabajador sin especializa- } \\
\text { ción }\end{array}$ & 7.7 & 19.4 & 40 & 13.9 & 667 & 19.9 \\
\hline & Trabajador especializado & 1598 & 43.9 & 122 & 42.4 & 1476 & 44.0 \\
\hline & Madre & & & & & & \\
\hline & $\begin{array}{l}\text { Trabajador es- } \\
\text { pecializado }\end{array}$ & 500 & 13.7 & 40 & 13.9 & 460 & 13.7 \\
\hline & $\begin{array}{l}\text { Trabajador sin especializa- } \\
\text { ción }\end{array}$ & 535 & 14.7 & 49 & 17.0 & 486 & 14.5 \\
\hline & $\begin{array}{l}\text { Sin trabajo re- } \\
\text { munerado }\end{array}$ & 1566 & 43.0 & 125 & 43.4 & 1441 & 42.9 \\
\hline
\end{tabular}




\begin{tabular}{|c|c|c|c|c|c|c|c|}
\hline \multirow[b]{2}{*}{ Nacionalidad } & \multicolumn{3}{|l|}{ Todos los Padres } & \multicolumn{2}{|c|}{$\begin{array}{l}\text { Padres de } \\
\text { niños con } \\
\text { NEAE }\end{array}$} & \multicolumn{2}{|c|}{$\begin{array}{l}\text { Padres de } \\
\text { niños sin } \\
\text { NEAE }\end{array}$} \\
\hline & Padre & & & & & & \\
\hline & Española & 2180 & 59.8 & 205 & 71.2 & 1975 & 58.8 \\
\hline & Otra & 1348 & 37.0 & 68 & 23.6 & 1280 & 38.1 \\
\hline & Madre & & & & & & \\
\hline & Española & 2188 & 60.0 & 199 & 69.1 & 1989 & 59.3 \\
\hline & Otra & 1379 & 37.8 & 80 & 27.8 & 1299 & 38.7 \\
\hline \multirow{2}{*}{$\begin{array}{l}\text { Tipo de centro } \\
\text { escolar }\end{array}$} & Publico & 2930 & 80.4 & 215 & 74.7 & 2715 & 80.9 \\
\hline & Concertado & 643 & 17.7 & 67 & 23.3 & 553 & 19.1 \\
\hline \multirow[t]{3}{*}{ Etapa } & E. Infantil & 802 & 22.0 & 37 & 12.8 & 765 & 22.8 \\
\hline & E. Primaria & 2080 & 57.1 & 190 & 66.0 & 1890 & 56.3 \\
\hline & ESO & 672 & 18.5 & 57 & 19.8 & 615 & 18.4 \\
\hline
\end{tabular}

\section{Instrumento}

El instrumento utilizado fue el elaborado por Hernández-Prados, Gomariz, Parra y García-Sanz (2017). Está compuesto por preguntas tanto de contenido referido a la implicación de las familias como de carácter sociodemográfico; fue aplicado en formato papel y se le facilitó a los equipos directivos y estos a los tutores de los centros quienes los remitieron a las familias de su alumnado. Responde al modelo teórico de partida, el instrumento cuenta con cinco valores de respuesta posibles en los que se permite identificar la frecuencia o intensidad con la que se da la participación de las familias en cada una de las siete dimensiones exploradas. El instrumento se validó por cinco profesores universitarios, por los equipos directivos de los catorce centros escolares y directivos de las AMPA del municipio de la Región de Murcia.

Una vez elaborados los cuestionarios dirigidos a las familias se tradujeron al árabe $\mathrm{y}$ al inglés con el fin de acceder a colectivos muy numerosos con esos idiomas, propio en un municipio con elevada tasa migratoria. La fiabilidad del instrumento, acotando las variables criterio referidas a las dimensiones de la participación, ha resultado ser muy satisfactoria (De Vellis, 2003), ya que se obtuvo un coeficiente Alpha de Cronbach con resultado de 0.966 .

\section{Procedimiento de recogida y análisis de datos}

Con el fin de conocer la realidad existente de forma objetiva y comprobable se ha realizado una descripción sistemática de hechos y características de una población, mediante un diseño de investigación cuantitativa, no experimental, exploratoria y de carácter evaluativo. El diseño de la investigación es transversal, queremos comprobar la relación entre variables en un momento determinado.

Los análisis de este estudio se han realizado utilizando el paquete estadístico SPSSv.24. Se ha utilizado estadística tanto descriptiva como inferencial, aplicando en este caso técnicas de contraste de carácter no paramétrico (Kruskal Wallis y U Mann Whitney), ya que al aplicar la prueba de Levene de homocedasticidad y prueba de normalidad 
de Kolmogorov-Smirnov, sus valores nos llevaron a tal consideración. Asimismo, se ha calculado la d de Cohen para considerar el tamaño del efecto. En todos los casos el nivel de significación estadístico obtenido fue de $\alpha=.05$.

\section{Resultados}

A continuación iremos exponiendo los resultados obtenidos de acuerdo con los objetivos propuestos.

\section{Objetivo 1}

Describir el grado de participación de las familias en la educación escolar, en relación a las dimensiones: comunicación con el centro, sentido de pertenencia e implicación en AMPA y CE, participación comunitaria y en la formación de las propias familias, distinguiendo entre las familias con hijos que presentan NEAE y aquellas cuyos hijos no presentan dichas necesidades.

Partiendo del modelo teórico señalado con anterioridad, y en los que se apoyan los instrumentos utilizados, la implicación de las familias en la educación escolar desde el hogar $(\mathrm{M}=4.16)$ es la dimensión en la que las familias consideran que participan en mayor medida, seguida del sentimiento de pertenencia $(M=3.64)$ y la comunicación con el centro $(\mathrm{M}=3.04)$. Respecto a las dimensiones que presentan una valoración media más baja, encontramos la participación comunitaria $(\mathrm{M}=2.29)$, seguida de la participación de las familias en las actividades organizadas por el centro $(\mathrm{M}=2.41)$. Podemos apreciar cómo en todas las dimensiones, a excepción de la Formación, las familias con hijos con NEAE obtienen medias superiores a las familias cuyos hijos no presentan dichas necesidades (ver Tabla 2).

Tabla 2.

Dimensiones de participación de las familias en la educación. Medias y desviaciones típicas en función de padres con hijos de NEAE y padres sin hijos de NEAE.

\begin{tabular}{lrrrrrr}
\hline & \multicolumn{2}{c}{ Todos los Padres } & $\begin{array}{c}\text { Padres de niños con } \\
\text { NEAE }\end{array}$ & \multicolumn{2}{c}{$\begin{array}{c}\text { Padres de niños sin } \\
\text { NEAE }\end{array}$} \\
\cline { 2 - 7 } & Media & $\begin{array}{c}\text { Desviación } \\
\text { Típica }\end{array}$ & Media & $\begin{array}{c}\text { Desviación } \\
\text { Típica }\end{array}$ & $\begin{array}{c}\text { Media } \\
\text { Desviación } \\
\text { Típica }\end{array}$ \\
\hline $\begin{array}{l}\text { Comunicación } \\
\text { con el centro }\end{array}$ & 3.04 & .68 & 3.17 & .67 & 3.04 & .68 \\
$\begin{array}{l}\text { Participación en } \\
\text { actividades del centro }\end{array}$ & 2.41 & .75 & 2.48 & .79 & 2.41 & .75 \\
$\begin{array}{l}\text { Sentimiento de } \\
\text { pertenencia }\end{array}$ & 3.64 & 1.07 & 3.84 & .92 & 3.62 & 1.08 \\
$\begin{array}{l}\text { Implicación } \\
\text { en el hogar }\end{array}$ & 4.16 & .61 & 4.22 & .56 & 4.16 & .62 \\
\hline
\end{tabular}




\begin{tabular}{lcccccc}
\hline & \multicolumn{2}{c}{ Todos los Padres } & $\begin{array}{c}\text { Padres de niños con } \\
\text { NEAE }\end{array}$ & \multicolumn{2}{c}{$\begin{array}{c}\text { Padres de niños sin } \\
\text { NEAE }\end{array}$} \\
\cline { 2 - 7 } & Media & $\begin{array}{c}\text { Desviación } \\
\text { Típica }\end{array}$ & Media & $\begin{array}{c}\text { Desviación } \\
\text { Típica }\end{array}$ & $\begin{array}{c}\text { Media } \\
\text { Desviación } \\
\text { Típica }\end{array}$ \\
\hline $\begin{array}{l}\text { Participación } \\
\text { en la AMPA }\end{array}$ & 2.86 & 1.04 & 2.93 & .99 & 2.86 & 1.04 \\
$\begin{array}{l}\text { Participación en } \\
\text { el Consejo Escolar }\end{array}$ & 2.71 & 1.14 & 2.83 & 1.10 & 2.70 & 1.14 \\
$\begin{array}{l}\text { Participación } \\
\text { Comunitaria }\end{array}$ & 2.29 & .91 & 2.34 & .88 & 2.29 & .91 \\
\begin{tabular}{l} 
Formación \\
\hline
\end{tabular} & 2.75 & .87 & 2.45 & .90 & 2.74 & .86 \\
\hline
\end{tabular}

\section{Objetivo 2}

En relación a la existencia de diferencias estadísticamente significativas en cada una de las dimensiones de participación exploradas, en función de la presencia de hijos/as con necesidades específicas de apoyo educativo se aplicó la prueba U Mann Whitney (Tabla 3).

Tabla 3.

Dimensiones de participación de las familias en la educación. Prueba U Mann Whitney en función de padres con hijos de NEAE y padres sin hijos de NEAE.

\begin{tabular}{|c|c|c|c|c|}
\hline Dimensiones & Grupo & $\begin{array}{c}\text { Rango } \\
\text { promedio }\end{array}$ & U Mann-Whitney & $\begin{array}{c}\text { Significación } \\
\text { asintótica }\end{array}$ \\
\hline \multirow{2}{*}{$\begin{array}{l}\text { Comunicación } \\
\text { con el centro }\end{array}$} & $\begin{array}{l}\text { Padres niños con } \\
\text { NEAE }\end{array}$ & 2018.97 & \multirow{2}{*}{405278.000} & \multirow{2}{*}{$.000^{*}$} \\
\hline & $\begin{array}{c}\text { Padres de niños sin } \\
\text { NEAE }\end{array}$ & 1772.39 & & \\
\hline \multirow{2}{*}{$\begin{array}{l}\text { Participación } \\
\text { en actividades } \\
\text { del centro }\end{array}$} & $\begin{array}{c}\text { Padres niños con } \\
\text { NEAE }\end{array}$ & 1850.35 & \multirow{2}{*}{437419.000} & \multirow{2}{*}{.162} \\
\hline & $\begin{array}{l}\text { Padres niños sin } \\
\text { NEAE }\end{array}$ & 1761.93 & & \\
\hline \multirow{2}{*}{$\begin{array}{l}\text { Sentimiento de } \\
\text { pertenencia }\end{array}$} & $\begin{array}{l}\text { Padres niños con } \\
\text { NEAE }\end{array}$ & 1970.55 & \multirow{2}{*}{420978.500} & \multirow{2}{*}{$.002^{*}$} \\
\hline & $\begin{array}{c}\text { Padres niños sin } \\
\text { NEAE }\end{array}$ & 1777.61 & & \\
\hline \multirow{2}{*}{$\begin{array}{l}\text { Implicación en } \\
\text { el hogar }\end{array}$} & $\begin{array}{l}\text { Padres niños con } \\
\text { NEAE }\end{array}$ & 1888.35 & \multirow{2}{*}{4484900.000} & \multirow{2}{*}{.134} \\
\hline & $\begin{array}{c}\text { Padres niños sin } \\
\text { NEAE }\end{array}$ & 1792.37 & & \\
\hline
\end{tabular}




\begin{tabular}{|c|c|c|c|c|}
\hline Dimensiones & Grupo & $\begin{array}{c}\text { Rango } \\
\text { promedio }\end{array}$ & U Mann-Whitney & $\begin{array}{c}\text { Significación } \\
\text { asintótica }\end{array}$ \\
\hline \multirow{2}{*}{$\begin{array}{l}\text { Participación } \\
\text { en la AMPA }\end{array}$} & $\begin{array}{c}\text { Padres niños con } \\
\text { NEAE }\end{array}$ & 1861.25 & \multirow{2}{*}{444516.500} & \multirow{2}{*}{.160} \\
\hline & $\begin{array}{c}\text { Padres niños sin } \\
\text { NEAE }\end{array}$ & 1772.35 & & \\
\hline \multirow{2}{*}{$\begin{array}{l}\text { Participación } \\
\text { en el Consejo } \\
\text { Escolar }\end{array}$} & $\begin{array}{c}\text { Padres niños con } \\
\text { NEAE }\end{array}$ & 1892.89 & \multirow{2}{*}{427077.000} & \multirow{2}{*}{$.038^{*}$} \\
\hline & $\begin{array}{c}\text { Padres niños sin } \\
\text { NEAE }\end{array}$ & 1761.51 & & \\
\hline \multirow{2}{*}{$\begin{array}{l}\text { Participación } \\
\text { Comunitaria }\end{array}$} & $\begin{array}{c}\text { Padres niños con } \\
\text { NEAE }\end{array}$ & 1851.02 & \multirow{2}{*}{448870.000} & \multirow{2}{*}{.255} \\
\hline & $\begin{array}{c}\text { Padres niños sin } \\
\text { NEAE }\end{array}$ & 1778.73 & & \\
\hline \multirow{2}{*}{ Formación } & $\begin{array}{c}\text { Padres niños con } \\
\text { NEAE }\end{array}$ & 1856.33 & \multirow{2}{*}{421166.500} & \multirow{2}{*}{.067} \\
\hline & $\begin{array}{c}\text { Padres niños sin } \\
\text { NEAE }\end{array}$ & 1741.30 & & \\
\hline
\end{tabular}

En la Tabla 3, aparecen diferencias estadísticamente significativas en las dimensiones Comunicación con el centro $(p=.000)$, Sentimiento de pertenencia $(p=.002)$ y Participación en el CE ( $p=.038)$. Las diferencias en las dimensiones Comunicación con el centro, Sentimiento de pertenencia y Participación en CE se presentan a favor de las familias que tienen hijos con NEAE frente a las familias cuyos hijos no tienen NEAE. Aunque considerando los valores obtenidos al analizar la d de Cohen $(-0.0337,0.0238$ y 0.0128 , respectivamente), entendemos que el tamaño del efecto no es suficientemente fuerte.

A continuación, presentamos específicamente cada una de las tres dimensiones en las que se presentan diferencias estadísticamente significativas, destacando aquellos ítems en los que aparecen dichas diferencias. La dimensión Comunicación con el centro se puede apreciar en la Tabla 4 .

Tabla 4.

Dimensión Comunicación con el centro. Prueba U Mann Whitney en función de padres con hijos de NEAE y padres sin hijos de NEAE.

\begin{tabular}{lcccc}
\hline Items & Grupo & $\begin{array}{c}\text { Rango } \\
\text { promedio }\end{array}$ & U Mann-Whitney & $\begin{array}{c}\text { Significación } \\
\text { asintótica }\end{array}$ \\
\hline $\begin{array}{l}\text { Asisto a las tutorías } \\
\begin{array}{l}\text { cuando me cita el } \\
\text { tutor de mi hijo }\end{array}\end{array}$ & $\begin{array}{c}\text { Padres niños con } \\
\text { NEAE }\end{array}$ & 1894.74 & & \\
\cline { 2 - 3 } & $\begin{array}{c}\text { Padres niños sin } \\
\text { NEAE }\end{array}$ & 1753.82 & 418531.500 & .008 \\
\hline
\end{tabular}




\begin{tabular}{|c|c|c|c|c|}
\hline Items & Grupo & $\begin{array}{c}\text { Rango } \\
\text { promedio }\end{array}$ & U Mann-Whitney & $\begin{array}{l}\text { Significación } \\
\text { asintótica }\end{array}$ \\
\hline \multirow{2}{*}{$\begin{array}{l}\text { Solicito tutorías a } \\
\text { lo largo del curso } \\
\text { académico }\end{array}$} & $\begin{array}{l}\text { Padres niños con } \\
\text { NEAE }\end{array}$ & 1975.92 & \multirow{2}{*}{384711.500} & \multirow{2}{*}{.000} \\
\hline & $\begin{array}{l}\text { Padres niños sin } \\
\text { NEAE }\end{array}$ & 1722.57 & & \\
\hline \multirow{2}{*}{$\begin{array}{l}\text { Mantengo } \\
\text { reuniones con el } \\
\text { resto de profesores } \\
\text { diferentes al tutor }\end{array}$} & $\begin{array}{l}\text { Padres niños con } \\
\text { NEAE }\end{array}$ & 1939.65 & \multirow{2}{*}{400827.500} & \multirow{2}{*}{.001} \\
\hline & $\begin{array}{l}\text { Padres niños sin } \\
\text { NEAE }\end{array}$ & 1732.21 & & \\
\hline \multirow{2}{*}{$\begin{array}{l}\text { Me reúno con } \\
\text { personas del equipo } \\
\text { directivo del centro }\end{array}$} & $\begin{array}{l}\text { Padres niños con } \\
\text { NEAE }\end{array}$ & 1861.27 & \multirow{2}{*}{418271.500} & \multirow{2}{*}{.021} \\
\hline & $\begin{array}{l}\text { Padres niños sin } \\
\text { NEAE }\end{array}$ & 1732.59 & & \\
\hline \multirow{2}{*}{$\begin{array}{l}\text { Me entrevisto con } \\
\text { el orientador y/o } \\
\text { especialistas en } \\
\text { educación especial } \\
\text { que atienden al } \\
\text { centro }\end{array}$} & $\begin{array}{l}\text { Padres niños con } \\
\text { NEAE }\end{array}$ & 2372.78 & \multirow[b]{2}{*}{263963.000} & \multirow[b]{2}{*}{.000} \\
\hline & $\begin{array}{l}\text { Padres niños sin } \\
\text { NEAE }\end{array}$ & 1671.14 & & \\
\hline \multirow{2}{*}{$\begin{array}{l}\text { Soy o he sido } \\
\text { representante de las } \\
\text { familias del grupo- } \\
\text { clase de mi hijo } \\
\text { para ayudar en la } \\
\text { comunicación con } \\
\text { el tutor }\end{array}$} & $\begin{array}{l}\text { Padres niños con } \\
\text { NEAE }\end{array}$ & 1576.44 & \multirow[t]{2}{*}{404653.500} & \multirow[t]{2}{*}{.000} \\
\hline & $\begin{array}{l}\text { Padres niños sin } \\
\text { NEAE }\end{array}$ & 1759.82 & & \\
\hline
\end{tabular}

Como podemos observar en la Tabla 4, aparecen diferencias estadísticamente significativas en los ítems asisto a las tutorías cuando me cita el tutor de mi hijo ( $p=.008)$, solicito tutorías a lo largo del curso académico $(p=.000)$, mantengo reuniones con el resto de profesores diferentes al tutor $(p=.001)$, me reúno con personas del equipo directivo del centro $(p=.021)$, me entrevisto con el orientador y/o especialistas en educación especial que atienden al centro $(p=.000)$. En todos estos ítems, las diferencias se presentan a favor de las familias que tienen hijos con NEAE frente a las familias que sus hijos no tienen NEAE. En el ítem referido a soy o he sido representante de las familias del grupo-clase de mi hijo para ayudar en la comunicación con el tutor $(p=.000)$, sucede al contrario, las diferencias se muestran a favor de las familias cuyos hijos no presentan NEAE.

La dimensión Sentimiento de Pertenencia se puede apreciar en la Tabla 5. 
Tabla 5.

Dimensión Sentimiento de Pertenencia. Prueba U Mann Whitney en función de padres con hijos de NEAE y padres sin hijos de NEAE.

\begin{tabular}{|c|c|c|c|c|}
\hline Items & Grupo & $\begin{array}{c}\text { Rango } \\
\text { promedio }\end{array}$ & U Mann-Whitney & $\begin{array}{l}\text { Significación } \\
\text { asintótica }\end{array}$ \\
\hline \multirow{2}{*}{$\begin{array}{l}\text { Me identifico con los } \\
\text { valores, ideas, actitudes, } \\
\text { metas, etc. del centro }\end{array}$} & $\begin{array}{c}\text { Padres niños } \\
\text { con NEAE }\end{array}$ & 1890.98 & \multirow[b]{2}{*}{420326.500} & \multirow[b]{2}{*}{.017} \\
\hline & $\begin{array}{l}\text { Padres niños } \\
\text { sin NEAE }\end{array}$ & 1750.23 & & \\
\hline \multirow{2}{*}{$\begin{array}{l}\text { Me siento miembro del } \\
\text { centro, lo considero algo } \\
\text { mío }\end{array}$} & $\begin{array}{c}\text { Padres niños } \\
\text { con NEAE }\end{array}$ & 1880.28 & \multirow{2}{*}{423856.000} & \multirow{2}{*}{.030} \\
\hline & $\begin{array}{l}\text { Padres niños } \\
\text { sin NEAE }\end{array}$ & 1749.48 & & \\
\hline \multirow{2}{*}{$\begin{array}{l}\text { Me siento atraído } \\
\text { hacia las actividades } \\
\text { o experiencias de } \\
\text { colaboración con las } \\
\text { familias que ofrece el } \\
\text { centro }\end{array}$} & $\begin{array}{l}\text { Padres niños } \\
\text { con NEAE }\end{array}$ & 1933.28 & \multirow{2}{*}{392985.000} & \multirow{2}{*}{.001} \\
\hline & $\begin{array}{l}\text { Padres niños } \\
\text { sin NEAE }\end{array}$ & 1729.31 & & \\
\hline \multirow{2}{*}{$\begin{array}{l}\text { Participar en el centro } \\
\text { educativo me hace sentir } \\
\text { que formo parte de él }\end{array}$} & $\begin{array}{l}\text { Padres niños } \\
\text { con NEAE }\end{array}$ & 1869.62 & \multirow{2}{*}{417953.000} & \multirow{2}{*}{.029} \\
\hline & $\begin{array}{l}\text { Padres niños } \\
\text { sin NEAE }\end{array}$ & 1738.46 & & \\
\hline \multirow{2}{*}{$\begin{array}{l}\text { Desde el inicio de la } \\
\text { escolarización de mi hijo } \\
\text { me he sentido acogido } \\
\text { e integrado por la } \\
\text { comunidad educativa }\end{array}$} & $\begin{array}{l}\text { Padres niños } \\
\text { con NEAE }\end{array}$ & 1896.24 & \multirow{2}{*}{426862.500} & \multirow{2}{*}{.020} \\
\hline & $\begin{array}{l}\text { Padres niños } \\
\text { sin NEAE }\end{array}$ & 1757.30 & & \\
\hline \multirow{2}{*}{$\begin{array}{l}\text { Me siento libre para } \\
\text { expresar en el centro } \\
\text { mis ideas, inquietudes, } \\
\text { sugerencias, quejas, etc. }\end{array}$} & $\begin{array}{l}\text { Padres niños } \\
\text { con NEAE }\end{array}$ & 1948.33 & \multirow{2}{*}{410427.000} & \multirow{2}{*}{.001} \\
\hline & $\begin{array}{l}\text { Padres niños } \\
\text { sin NEAE }\end{array}$ & 1752.25 & & \\
\hline \multirow{2}{*}{$\begin{array}{l}\text { Recomendaría a otras } \\
\text { personas con hijos en } \\
\text { edad escolar el centro } \\
\text { educativo al que asiste } \\
\text { mi hijo }\end{array}$} & $\begin{array}{l}\text { Padres niños } \\
\text { con NEAE }\end{array}$ & 1899.89 & \multirow{2}{*}{426228.500} & \multirow{2}{*}{.022} \\
\hline & $\begin{array}{l}\text { Padres niños } \\
\text { sin NEAE }\end{array}$ & 1763.08 & & \\
\hline \multirow{2}{*}{$\begin{array}{l}\text { El centro facilita que me } \\
\text { sienta vinculado a él }\end{array}$} & $\begin{array}{c}\text { Padres niños } \\
\text { con NEAE }\end{array}$ & 1909.52 & \multirow{2}{*}{418483.500} & \multirow{2}{*}{.011} \\
\hline & $\begin{array}{l}\text { Padres niños } \\
\text { sin NEAE }\end{array}$ & 1755.18 & & \\
\hline
\end{tabular}


Cuando observamos la Tabla 5, aparecen diferencias estadísticamente significativas en los Ítems me identifico con los valores, ideas, actitudes, metas, etc. del centro ( $\mathrm{p}=.017)$, me siento miembro del centro, lo considero algo mío ( $\mathrm{p}=.030)$, me siento atraído hacia las actividades o experiencias de colaboración con las familias que ofrece el centro $(\mathrm{p}=.001)$, participar en el centro educativo me hace sentir que formo parte de él $(\mathrm{p}=.029)$, desde el inicio de la escolarización de mi hijo me he sentido acogido e integrado por la comunidad educativa $(\mathrm{p}=.020)$, me siento libre para expresar en el centro mis ideas, inquietudes, sugerencias, quejas, etc. $(\mathrm{p}=.001)$, recomendaría a otras personas con hijos en edad escolar el centro educativo al que asiste mi hijo ( $p=.022)$, el centro facilita que me sienta vinculado a él ( $\mathrm{p}=.011)$. Las diferencias en los Ítems se presentan a favor de las familias que tienen hijos con NEAE frente a las familias que sus hijos no tienen NEAE.

Podemos observar la dimensión implicación en el CE en la Tabla 6.

Tabla 6.

Dimensión implicación en el Consejo Escolar. Prueba U Mann Whitney en función de padres con hijos de NEAE y padres sin hijos de NEAE.

\begin{tabular}{|c|c|c|c|c|}
\hline Items & Grupo & $\begin{array}{c}\text { Rango } \\
\text { promedio }\end{array}$ & U Mann-Whitney & $\begin{array}{c}\text { Significación } \\
\text { asintótica }\end{array}$ \\
\hline \multirow{2}{*}{$\begin{array}{l}\text { Sé lo que es el } \\
\text { Consejo Escolar } \\
\text { del centro }\end{array}$} & $\begin{array}{c}\text { Padres niños } \\
\text { con NEAE }\end{array}$ & 1869.11 & \multirow{2}{*}{403620.500} & \multirow{2}{*}{.017} \\
\hline & $\begin{array}{l}\text { Padres niños } \\
\text { sin NEAE }\end{array}$ & 1723.41 & & \\
\hline \multirow{2}{*}{$\begin{array}{l}\text { Conozco las } \\
\text { funciones de los } \\
\text { representantes del } \\
\text { Consejo Escolar } \\
\text { del centro }\end{array}$} & $\begin{array}{l}\text { Padres niños } \\
\text { con NEAE }\end{array}$ & 1850.58 & \multirow[b]{2}{*}{417223.000} & \multirow[b]{2}{*}{.057} \\
\hline & $\begin{array}{c}\text { Padres niños } \\
\text { sin NEAE }\end{array}$ & 1733.64 & & \\
\hline \multirow{2}{*}{$\begin{array}{l}\text { Estoy informado } \\
\text { sobre el calendario } \\
\text { o proceso de } \\
\text { las elecciones a } \\
\text { Consejo Escolar } \\
\text { del centro }\end{array}$} & $\begin{array}{c}\text { Padres niños } \\
\text { con NEAE }\end{array}$ & 1766.24 & \multirow[b]{2}{*}{415403.500} & \multirow[b]{2}{*}{.052} \\
\hline & $\begin{array}{l}\text { Padres niños } \\
\text { sin NEAE }\end{array}$ & 1730.11 & & \\
\hline \multirow{2}{*}{$\begin{array}{l}\text { Participo en las } \\
\text { elecciones al } \\
\text { Consejo Escolar }\end{array}$} & $\begin{array}{c}\text { Padres niños } \\
\text { con NEAE }\end{array}$ & 1847.70 & \multirow{2}{*}{408733.000} & \multirow{2}{*}{.042} \\
\hline & $\begin{array}{l}\text { Padres niños } \\
\text { sin NEAE }\end{array}$ & 1726.39 & & \\
\hline
\end{tabular}

Al observar la Tabla 6, vemos que aparecen diferencias estadísticamente significativas en los ítems sé lo que es el Consejo Escolar del centro ( $p=.017)$, y participo en 
las elecciones al CE ( $p=.042)$. Las diferencias en los Ítems se presentan a favor de las familias que tienen hijos con NEAE frente a las familias que sus hijos no tienen NEAE.

\section{Objetivo 3}

Respecto al tercer objetivo relativo a estudiar la significación estadística en las dimensiones de participación familiar, teniendo en cuenta la procedencia nacional de los progenitores (no española o española), en función de si su hijo posee o no NEAE. Hemos considerando la procedencia familiar no española si, al menos, la de uno de los progenitores es extranjera. En general, las familias de origen no español presentan una participación media global de $2.76(\mathrm{DT}=.633)$, mientras que las familias de origen español obtienen una media situada en 3.21 (DT=.508), siendo el tamaño del efecto d de Cohen de .7876 , considerado como relevante.

Una vez segmentado el archivo, en el caso de los resultados para familias con alguno de sus progenitores de origen no español, aplicada la prueba U Mann Whitney, en función de tener hijo con NEAE o no, encontramos que no se aprecian diferencias estadísticamente significativas en ninguna de las dimensiones exploradas relativas a la participación entre las familias no españolas con hijos con NEAE y aquellas cuyos hijos no tienen NEAE (Comunicación $p=.123$; Actividades centro $p=.350$, Sentimiento pertenencia $p=.116$, Implicación desde el hogar $p=.809$, AMPA $p=.789$, CE $\mathrm{p}=.361$, Comunitaria $p=.603$ y Formación $p=.680$ ).

Mientras, en el caso de familias de origen español, en función de si sus hijos poseen NEAE o no, presentamos a continuación los resultados en la Tabla 7.

Tabla 7.

Dimensiones de participación de las familias en la educación. Prueba U Mann Whitney en función de si tienen hijos con NEAE. Familias de origen español.

\begin{tabular}{|c|c|c|c|c|}
\hline Dimensiones & Grupo & $\begin{array}{l}\text { Rango } \\
\text { promedio }\end{array}$ & U Mann-Whitney & $\begin{array}{l}\text { Significación } \\
\text { asintótica }\end{array}$ \\
\hline \multirow{2}{*}{$\begin{array}{l}\text { Comunicación con } \\
\text { el centro }\end{array}$} & $\begin{array}{l}\text { Padres niños con } \\
\text { NEAE }\end{array}$ & 1252.96 & \multirow{2}{*}{148606.000} & \multirow{2}{*}{$.000^{*}$} \\
\hline & $\begin{array}{c}\text { Padres niños sin } \\
\text { NEAE }\end{array}$ & 1030.55 & & \\
\hline \multirow{2}{*}{$\begin{array}{l}\text { Participación en } \\
\text { actividades del } \\
\text { centro }\end{array}$} & $\begin{array}{l}\text { Padres niños con } \\
\text { NEAE }\end{array}$ & 1086.06 & \multirow{2}{*}{174331.000} & \multirow{2}{*}{.227} \\
\hline & $\begin{array}{l}\text { Padres niños sin } \\
\text { NEAE }\end{array}$ & 1031.88 & & \\
\hline \multirow{2}{*}{$\begin{array}{l}\text { Sentimiento de } \\
\text { pertenencia }\end{array}$} & $\begin{array}{c}\text { Padres niños con } \\
\text { NEAE }\end{array}$ & 1089.41 & \multirow{2}{*}{182401.500} & \multirow{2}{*}{.373} \\
\hline & $\begin{array}{l}\text { Padres niños sin } \\
\text { NEAE }\end{array}$ & 1049.20 & & \\
\hline
\end{tabular}




\begin{tabular}{|c|c|c|c|c|}
\hline Dimensiones & Grupo & $\begin{array}{c}\text { Rango } \\
\text { promedio }\end{array}$ & U Mann-Whitney & $\begin{array}{c}\text { Significación } \\
\text { asintótica }\end{array}$ \\
\hline \multirow{2}{*}{$\begin{array}{l}\text { Implicación en el } \\
\text { hogar }\end{array}$} & $\begin{array}{l}\text { Padres niños con } \\
\text { NEAE }\end{array}$ & 1052.52 & \multirow{2}{*}{189550.500} & \multirow{2}{*}{.952} \\
\hline & $\begin{array}{l}\text { Padres niños sin } \\
\text { NEAE }\end{array}$ & 1055.26 & & \\
\hline \multirow{2}{*}{$\begin{array}{l}\text { Participación en la } \\
\text { AMPA }\end{array}$} & $\begin{array}{l}\text { Padres niños con } \\
\text { NEAE }\end{array}$ & 1033.23 & \multirow{2}{*}{185712.000} & \multirow{2}{*}{.717} \\
\hline & $\begin{array}{l}\text { Padres niños sin } \\
\text { NEAE }\end{array}$ & 1049.55 & & \\
\hline \multirow{2}{*}{$\begin{array}{l}\text { Participación en el } \\
\text { Consejo Escolar }\end{array}$} & $\begin{array}{l}\text { Padres niños con } \\
\text { NEAE }\end{array}$ & 1046.64 & \multirow{2}{*}{188382.000} & \multirow{2}{*}{.993} \\
\hline & $\begin{array}{l}\text { Padres niños sin } \\
\text { NEAE }\end{array}$ & 1047.04 & & \\
\hline \multirow{2}{*}{$\begin{array}{l}\text { Participación } \\
\text { Comunitaria }\end{array}$} & $\begin{array}{l}\text { Padres niños con } \\
\text { NEAE }\end{array}$ & 1042.37 & \multirow{2}{*}{187531.500} & \multirow{2}{*}{.861} \\
\hline & $\begin{array}{l}\text { Padres niños sin } \\
\text { NEAE }\end{array}$ & 1050.25 & & \\
\hline \multirow{2}{*}{ Formación } & $\begin{array}{l}\text { Padres niños con } \\
\text { NEAE }\end{array}$ & 1112.79 & \multirow{2}{*}{168279.000} & \multirow{2}{*}{$.048^{*}$} \\
\hline & $\begin{array}{l}\text { Padres niños sin } \\
\text { NEAE }\end{array}$ & 1024.59 & & \\
\hline
\end{tabular}

En la Tabla 7, podemos apreciar que en dos de las dimensiones de la participación existen diferencias estadísticamente significativas, Comunicación $(p=.000)$ y Formación ( $p=.048)$ a favor de los padres de hijos con NEAE, lo que muestra que estas familias se comunican con mayor frecuencia con el centro, así como se autoperciben como más formadas en relación a las familias de origen español cuyos hijos no poseen NEAE.

\section{Discusión y Conclusiones}

En general, podemos afirmar que las familias con hijos con NEAE escolarizados en las etapas educativas desde Educación Infantil a Educación Secundaria Obligatoria participan globalmente de manera más frecuente e intensa en la educación escolar de sus hijos que aquellas familias cuyos hijos no presentan dichas necesidades educativas, lo cual se aprecia en todas las dimensiones de la implicación parental exploradas, a excepción de la formación a familias.

Coincidimos con Robledo y García-Sánchez (2014) respecto a la mayor implicación parental que atribuyen a los padres de alumnos que presentan TDAH, aunque en este estudio también se concluye que las familias cuyos hijos presentan DEA (Dificultades Específicas de Aprendizaje) se implican significativamente menos en los procesos de enseñanza-aprendizaje que las familias cuyos hijos presentan TDAH. Además, apuntan como argumento para justificar estas diferencias que las necesidades educativas del hijo son más evidentes y hace que los padres las detecten con 
anterioridad, estén más alerta y proactivos respecto a la implicación en su proceso educativo que cuando las dificultades específicas de aprendizaje son menos evidentes y pueden pasar más desapercibidas, por su menor repercusión también en el proceso de enseñanza y aprendizaje.

Se hace preciso, profundizar en estudios de carácter más pormenorizado que consideren cada una de las dificultades específicas de aprendizaje o necesidades educativas del alumnado como una de las variables a contemplar en la participación familiar en la educación escolar.

Podemos concluir que las familias cuyos hijos tienen NEAE, manifiestan tener una comunicación más frecuente y fluida con el centro educativo y el profesorado, indican poseer un sentimiento de pertenencia a la institución escolar más elevado, manifiestan implicarse formalmente a través del CE del centro de manera más activa, en contraste con las familias cuyos hijos no presentan NEAE.

Respecto a la comunicación con el centro, es entendible que las familias cuyos hijos presentan necesidades educativas contacten de manera más habitual y asistan a reuniones, tanto grupales, como individuales, de manera más frecuente a lo que suele hacerlo el resto de familias, bien por iniciativa propia, o bien a petición del profesorado, especialistas u orientador educativo. Este contacto y comunicación más frecuente se entiende en el contexto de intentar ofrecer una respuesta educativa lo más consensuada posible antes las dificultades de aprendizaje que presenta este alumnado. En nuestra opinión, y de acuerdo con otros autores (Bonell, 2016; Macia, 2018), el profesorado debe cambiar la mirada y diseñar estrategias comunicativas más enriquecedoras que desde la necesidad o urgencia de comunicar con las familias ante situaciones de carácter terapéutico o correctivo, avancen hacia fórmulas de colaboración y formación a familias para diseñar cooperativamente las respuestas educativas más acertadas para el niño, desde la escuela y desde el hogar. Desde los centros se debe intentar mantener informada a la familia sobre los diferentes cauces que pueden seguir para implicarse y participar en los centros escolares. Al mismo tiempo desde las familias se debe intentar el uso de los cauces facilitados para dar a conocer a los centros cuáles son sus necesidades de comunicación y participación, apostando por modelos de colaboración conjunta entre profesorado y familias (Bonell, 2016).

En relación al sentimiento de pertenencia, compartimos con Hernández-Prados, Gomariz, Parra y García-Sanz (2015) que este elemento es motivador de la participación familiar en la escuela, actuando como potenciador de la misma y, a su vez, como consecuencia de dicha implicación, y este sentimiento se da cuando la escuela se convierte en comunidad que acoge. Consideramos que las familias participantes en nuestro estudio se sienten, en general, acogidas y bienvenidas por sus centros escolares. Este sería el clima de escuela realmente acogedora, inclusiva, en la que todos somos bien recibidos, según Ainscow (2001). Consideramos que los centros escolares, y especialmente los equipos directivos y los docentes como principales facilitadores (Andrés y Giró, 2016), han de estimular en toda la diversidad de familias que los forman, su sentido de pertenencia al centro educativo, en general, al grupo-clase en particular, y a la comunidad educativa, lo que se puede convertir en un punto clave para facilitar diversas maneras y vías de participar en la vida escolar. 
En cuanto a la implicación en el Consejo Escolar, el trabajo previo de Parra et al. (2014b) ya nos avanza que esta dimensión de la participación, referida a su vertiente más normativa y de carácter colectivo, se sitúa en niveles por debajo de lo deseable, considerando a las familias en general, aunque asciende bastante si analizamos la situación de las familias cuyos hijos están escolarizados en centros específicos de Educación Especial. Quizá esta mayor participación en el Consejo Escolar a favor de las familias cuyos hijos presentan necesidades educativas, detectada en nuestro estudio, esté vislumbrando que estas familias pueden mostrar un mayor compromiso ciudadano a través de estas vías colectivas de participación, aunque su mayor implicación pasa por estar informadas del CE, participar significativamente más en las elecciones, que realmente formar parte de este órgano, que ciertamente en los últimos tiempos se ha convertido más en órgano consultivo que decisorio en los centros educativos escolares.

En concreto, cuando diferenciamos entre familias de origen no español y familias españolas, las dimensiones que arrojan una mayor participación de las familias españolas con hijos con NEAE son las referidas a comunicación y a formación. La nacionalidad no aporta una mayor o menor participación escolar significativa. Tan sólo distinguimos que entre las familias de origen español, las que poseen hijos con NEAE afirman comunicarse más con el centro y se valoran con un mayor nivel de formación, que aquellas familias cuyos hijos no poseen NEAE. En esta línea, López-Pascual y Carmona (2018) afirman que las familias muestran una "satisfacción generalizada acerca de la comunicación y cooperación que se lleva a cabo entre los profesionales del ámbito educativo y las familias con hijos con diversidad funcional" (p.94). Sin embargo, nos encontramos en un contexto en el que los padres y madres de origen no español, independientemente de tener hijos con NEAE, no muestran niveles elevados de participación, lo que suele ocurrir, según Giró, Mata, Vallespir y Vigo (2014) en los centros con un elevado nivel de escolarización de alumnado extranjero, debido quizá a los obstáculos culturales y lingüísticos.

Por todo ello, es preciso abordar la participación de las familias desde una perspectiva sociocomunitaria, abriendo nuevas opciones al trabajo colectivo y cooperativo entre familias y escuelas, padres y madres y docentes (Ballesteros-Velázquez, Aguado-Odina y Malik-Liévano, 2014; San Fabián, 2005) e impulsando que las instituciones permitan la inclusión de las familias de origen no español, permitiendo una educación de calidad y con equidad para todos los estudiantes (Hernández-Prados et al., 2016). Que familias y profesorado creen dinámicas para compartir objetivos educativos colaborativamente siempre redundará en el bien de los hijos, del alumnado, más allá de la mejora del rendimiento, la disminución del abandono escolar o la mejora del clima de convivencia. El crecimiento y la suma es para toda la comunidad educativa: se enriquecen los centros educativos, el clima educativo de las familias y la conformación personal de sus miembros. La inclusión supone también eliminar muros, barreras y fronteras entre las personas, entre profesorado y familias, viviendo la diversidad como propio de la normalidad. El cambio de mirada de unos a otros es la causa y la consecuencia de caminar juntos hacia una misma meta. 


\section{Referencias}

Ainscow, M. (2001) Desarrollo de escuelas inclusivas. Madrid: Narcea.

Alomar, B. (2006). Personal and family paths to pupil achievement. Social Behavior and Personality, 34(8), 907-922. DOI: https://doi.org/10.2224/sbp.2006.34.8.907

Andrés, S. y Giró, J. (2016). El papel y la representación del profesorado en la participación de las familias en la escuela. Revista Electrónica Interuniversitaria de Formación del Profesorado, 19(1), 61-71. DOI: http://dx.doi.org/10.6018/reifop.19.1.245461

Ballesteros-Velázquez, B., Aguado-Odina, T. y Malik-Liévano, B. (2014). Escuelas para todos: diversidad y educación obligatoria. Revista Electrónica Interuniversitaria de Formación del Profesorado, 17(2), 93-107. DOI: http://dx.doi.org/10.6018/reifop.17.2.197351

Bonell, L. (2016). La promoción de la participación educativa de las familias en la escuela desde una perspectiva transformadora: un estudio de casos (Tesis doctoral). Universidad de Valladolid. Recuperado de : https:/uvadoc.uva.es/bitstream/handle/10324/16723/ Tesis966160411.pdf;jsessionid=3E7ED2CCA8831101CA09438D5605100A?sequence=1

Castro, M., Expósito, E., Lizasoain, L., López, E. y Navarro, E. (2014). Participación familiar y rendimiento académico. Una síntesis meta-analítica. En Consejo Escolar del Estado (Coord.), La participación de las familias en la educación escolar (pp. 83-105). Madrid: Ministerio de Educación, Cultura y Deporte.

Chang-Hun, C. H. \& Juyoung Song, J. (2012). Functions of parental involvement and effects of school climate on bullying behaviors among South Korean middle school students. Journal of interpersonal violence, 27(12), 2437-2464. DOI: https://doi. org/10.1177/0886260511433508.

Cox, D.D. (2005). Evidence based interventions using home-school collaboration. School Psychology Quarterly, 20(4), 473-97. DOI: https://doi.org/10.1521/scpq.2005.20.4.47

Darling-Hammond, L. (2000). Teacher quality and student achievement a review of state policy evidence. Education policy analysis archives, 8(1). Recuperado de https:// epaa.asu.edu/ojs/article/viewFile/392/515

De Vellis, R.F. (2003). Scale development: Theory and applications ( $2^{\mathrm{a}}$ ed.), Thousand Oaks, CA: Sage.

Egido, I. (2014). Marcos normativos de la participación de las familias en los sistemas educativos europeos. Una visión comparada. En Consejo Escolar del Estado (Coord.), La participación de las familias en la educación escolar (pp.35-56). Madrid: Ministerio de Educación Cultura y Deporte.

Epstein, J.L. (2002). School, Family, and Community Partnerships: Caring for the children we share. En J.L. Epstein (Ed.), Family, and Community Partnerships: Your Handbook for Action (pp. 7-29). California, CORWIN PRESS, INC.

Garreta, J. (2015). La comunicación familia-escuela en Educación Infantil y Primaria. Revista de la Asociación de Sociología de la Educación, 8(1), 71-85.

Giró, J., Mata, A., Vallespir, J. y Vigo, B. (2014). Familias y escuelas: los diferentes discursos sobre la participación. Ehquidad International Welfare Policies and Social Work Journal, 2, 65-89.

Gomariz, M.A., Parra, J., García-Sanz, M.P. y Hernández-Prados, M.A. (2019). De lo formal a lo real. Análisis de la participación familiar en Asociaciones de madres y 
padres y Consejos escolares. Aula Abierta, 48(1), 85-96. DOI: https://doi.org/10.17811/ rifie.48.1.2019.85-96

Hernández-Prados, M.A., García-Sanz, M.A. Parra, J. y Gomariz, M.A. (2017). Involvement of immigrant families in the school life. Social and Behavioral Sciences, 237, 157-163. DOI: https://doi.org/10.1016/j.sbspro.2017.02.057

Hernández-Prados, M.A., García-Sanz, M.P., Gomariz, M.A., y Parra, J. (2019). Perfiles de participación familiar en Educación Secundaria Obligatoria. Anales de Psicología. 35(1), 84-94. DOI: http://dx.doi.org/10.6018/analesps.35.1.325981

Hernández-Prados, M.A., Gomariz, M.A., Parra, J. y García-Sanz, M.P. (2015). El sentimiento de pertenencia en la relación entre familia y escuela. Participación Educativa. Revista del Consejo Escolar del Estado, 4(7), 49-57.

Hernández-Prados, M.A., Gomariz, M.A., Parra, J. y García-Sanz, M.P. (2016). Familia, inmigración y comunicación con el centro escolar: Un estudio comparativo. Educación XXI, 19(2), 127-151. DOI: https://doi.org/10.5944/educXX1.19.2

Hernández-Prados, M.A., Gomariz, M.A., Parra, J. y García-Sanz, M.P. (2017). Valoración de la participación de las familias en la educación de los hijos. Cuestionario a familias. Recuperado de http://hdl.handle.net/10201/53821

Ley Orgánica de Educación 2/2006 de 3 de mayo. BOE no 106, de 4 de mayo. Madrid. Recuperado de https://www.boe.es/eli/es/lo/2006/05/03/2/con

Ley Orgánica para la Mejora de la Calidad Educativa 8/2013 de 9 de diciembre. BOE $\mathrm{n}^{\mathrm{o}}$ 295, de 10 de diciembre. Madrid. Recuperado de https://www.boe.es/eli/es/ lo/2013/12/09/8/con

López-Pascual, G. y Carmona, C. (2018). La inclusión socio-educativa de niños y jóvenes con diversidad funcional: perspectiva de las familias. Revista Nacional e Internacional de Educación Inclusiva, 11(2), 83-98.

Macia, M. (2018). Aproximación teórica a la comunidad familia-escuela. Estrategias de mejora. Ehquidad International Welfare Policies and Social Work Journal, 10.

Parra, J., García-Sanz, M. P., Gomariz, M. A., y Hernández-Prados, M. A. (2014a). Perfiles de participación de las familias españolas en los centros educativos. En Consejo Escolar del Estado (Coord.), La participación de las familias en la educación escolar (pp. 127-148). Madrid: Ministerio de Educación, Cultura y Deporte.

Parra, J., García-Sanz, M. P., Gomariz, M. A., y Hernández-Prados, M. A. (2014b). Implicación de las familias en los consejos escolares de los centros. En Consejo Escolar del Estado (Coord.), La participación de las familias en la educación escolar (pp. 127-148). Madrid: Ministerio de Educación, Cultura y Deporte.

Pascale, L.D., Carrión Martínez, J.J., y Fernández Martínez, M.M. (2017). El estatus y roles de la familia en la escuela inclusiva en Italia: el caso de un instituto profesional. Revista de Educación Inclusiva, 10(2), 181-194.

Peñafiel, F. (2012). Planteamiento inclusivo de la participación de la familia en contextos de intervención con alumnos con discapacidad. Revista de Educación Inclusiva, $5(2), 117-127$.

Robledo, P. y García-Sánchez, J.N. (2014). Contexto familiar del alumnado con dificultades de aprendizaje o TDAH, percepciones de padres e hijos. Estudios sobre Educación, 26, 149-173. 
San Fabián, J.L. (2005). La participación de los centros escolares como ejercicio de la ciudadanía.. X Congreso Internacional de Educación Familiar. Dpto. de educación de la Universidad de La Palmas de Gran Canaria, 16-19 de marzo.

Saracostti, M., Lara, L., Martella, D., Miranda, H., Miranda-Zapata, E.D. \& Reininger, T. (2019). Influence of Family Involvement and Children's Socioemotional Development on the Learning Outcomes of Chilean Students. Frontiers in Psychology. Educational Psychology. 28 february. DOI: https://doi.org/10.3389/fpsyg.2019.00335

Vogels, R. (2002). Oudersbij de Les. Betrokkenheid van Oudersbij de School van hun Kind. Den Haag: Sociaal Cultureel Planbureau.

Fecha de recepción: 1 de julio de 2019.

Fecha de revisión: 29 de julio de 2019.

Fecha de aceptación: 21 de enero de 2020. 